\title{
¿Músculo Digástrico o Músculo Digastricomastoideo?
}

\author{
Digastric Muscle or Digastricmastoid Muscle?
}

Pamela Carvallo $^{1,2}$; Eugenia Carvallo ${ }^{1,3}$ \& Mariano del Sol ${ }^{1,4}$

CARVAllo, P.; CARVAllo, E. \& DEL SOL, M. ¿Músculo digástrico o músculo digastricomastoideo? Int. J. Morphol., 35(1):368-370, 2017.

RESUMEN: El músculo digástrico aparece mencionado en Terminología Anatomica con el código A04.2.03.006, pertenece al grupo de los músculos suprahioideos y está formado por dos vientres (latín: gaster) unidos por un tendón intermedio. El vientre anterior se origina en la fosa digástrica de la mandíbula y el vientre posterior en la incisura mastoidea del hueso temporal, ambos se insertan a través de una banda fibrosa, derivada de la capa pretraqueal de la fascia cervical profunda en el cuerpo y cuerno mayor del hueso hioideo. Los vientres anterior y posterior se originan del primer y segundo arco branquial respectivamente, siendo inervado el vientre anterior por el nervio milohioideo y el vientre posterior por el nervio facial. En su denominación el músculo digástrico hace referencia al origen del vientre anterior y a la clasificación muscular según forma, sin mencionar el origen de su vientre posterior. Es nuestro objetivo revisar este término y recomendar incluir en el nombre el origen "mastoideo" del músculo digástrico, denominándolo músculo digastricomastoideo. Este cambio se basa en el origen embriológico e inervación diferentes de ambos vientres y se relaciona con los objetivos propuestos por Terminologia Anatomica, que recomienda nombres descriptivos, informativos y armónicos con las estructura óseas relacionadas, favoreciendo la comunicación científica y la enseñanza-aprendizaje de la morfología.

PALABRAS CLAVE: Músculo Digástrico; Músculo Digastricomastoideo; Terminología Anatomica.

\section{INTRODUCCIÓN}

El músculo digástrico aparece mencionado en Terminologia Anatomica con el código A04.2.03.006, pertenece al grupo de los músculos suprahioideos y está formado por dos vientres (latín: gaster) unidos por un tendón intermedio (Moore \& Dalley, 2007).

El vientre anterior se origina en la fosa digástrica de la mandíbula y el vientre posterior en la incisura mastoidea del hueso temporal, ambos se insertan a través de una banda fibrosa, derivada de la lámina pretraqueal en el cuerpo y cuerno mayor del hueso hioides (Testut \& Latarjet, 1979; Rouvière \& Delmas, 2002; Moore \& Dalley; Platzer, 2008; Shünke $e t$ al ., 2011). Está localizado en la región cervical anterior (Fig. 1). Sus vientres limitan los triángulos submental, submandibular y carotídeo (Testut \& Latarjet; Liquidate, et al., 2007; Moore \& Dalley; Platzer; Shünke et al.,).

El triángulo submandibular, esta delimitado por los vientres anterior y posterior del músculo digástrico y el margen inferior de la mandíbula y contiene: la glándula salival submandibular, linfonodos submandibulares, arteria y vena facial, nervio hipogloso y nervio milohioideo (Testut \& Latarjet; Liquidate, et al.,; Moore \& Dalley; Platzer; Shünke et al.,).

Por su origen embriológico, los vientres anterior y posterior del músculo digástrico se originan del primer y segundo arco faríngeo respectivamente, siendo inervado el vientre anterior por el nervio milohioideo y el vientre posterior por el nervio facial. Su función es actuar como depresor de la mandíbula, elevador y retractor del hueso hioideo durante la deglución y flexor accesorio de cabeza y cuello por su vientre anterior (Testut \& Latarjet; Liquidate, et al.,; Moore \& Dalley; Platzer; Shünke et al.,)

Las variaciones anatómicas de este músculo pueden ser tanto unilaterales como bilaterales y se presentan con mayor frecuencia en el vientre anterior del músculo digástrico y consisten en diferencias en la forma e inserción muscular (Liquidate et al.; Mascaro et al., 2011; Kalniev et al., 2013;

\footnotetext{
${ }^{1}$ Doctorado en Ciencias Morfológicas, Universidad de La Frontera, Temuco, Chile.

${ }^{2}$ Universidad Católica de Temuco, Temuco, Chile.

${ }^{3}$ Universidad Mayor, Sede Temuco, Chile.

${ }^{4}$ Centro de Investigación en Ciencias Biomédicas, Universidad Autónoma de Chile, Chile.
} 
Das et al., 2014). Según Bergman et al. (2017) un vientre anterior atípico del músculo digástrico fue encontrado en 6,6\% de los casos por Hallett (1847), en 5,9\% por Wood (1862), en $51 \%$ por Bianchi (1890), en 6,0\% por Le Double (1891), en $12 \%$ por Loth, en $26 \%$ por Bavero (1896), en $36 \%$ (fetal) por Bianchi (1896), en $48 \%$ por Stracher (1908), en $52 \%$ por Ruffini (1908), en 52,7\% por Stracker (1928), en 63,3\% por Zalbek (1933) y en $73 \%$ por Bertalli (1927).

El vientre posterior del músculo digástrico se puede originar de la incisura mastoides o de la parte lateral de la línea nucal superior. Ocasionalmente se fusiona con el músculo estilohioideo, ambos se forman partir de la misma masa común del mesénquima y puede que no se dividan correctamente. Si el vientre anterior del músculo digástrico no se diferencia del estilohioideo, el vientre posterior se puede originar del ángulo de la mandíbula y no del hioides. Se ha descrito un caso de "músculo digástrico" sin conección entre su vientre anterior y posterior, originándose el vientre anterior de la fosa digástrica e insertándose en el cuerpo del hueso hioides, mientras que el vientre posterior se originaba de la incisura mastoides y se insertaba en el proceso estiloides (Bergman et al.).

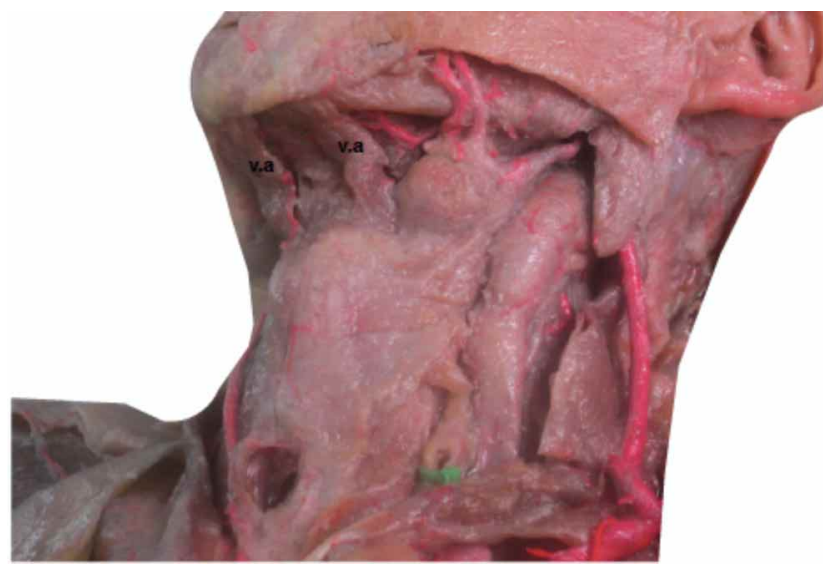

Fig. 1. Vientre anterior músculo digástrico derecho e izquierdo.

\section{FUNDAMENTACIÓN}

Una de las recomendaciones de Terminologia Anatomica es que la nomenclatura de las estructuras anatómicas sea descriptiva, fácil de recordar y armonizada con las estructuras vecinas. Se ha afirmado que la terminología científico-técnica se caracteriza por ser unívoca y concisa, para lograr la precisión conceptual requerida por la comunicación especializada (Whitmore, 1999; Cabré Castellví, 2003; Jiménez Gutiérrez, 2009; Edo Marza, 2012). En general los músculos suprahioideos son denominados según sus dos orígenes, siendo la única excepción el músculo digástrico.

\section{CONCLUSIÓN}

En su denominación el músculo digástrico hace referencia al origen del vientre anterior y a la clasificación muscular según forma, sin mencionar el origen de su vientre posterior. Fue nuestro objetivo revisar éste término y recomendar incluir en el nombre el origen "mastoideo" del músculo digástrico, denominándolo músculo digastricomastoideo. Este cambio se basa en el origen embriológico e inervación diferentes de ambos vientres y se relaciona con los objetivos propuestos por Terminologia Anatomica, que recomienda nombres descriptivos, informativos y armónicos con las estructura óseas relacionadas, favoreciendo la comunicación científica y la enseñanza-aprendizaje de la morfología.

CARVAllo, P.; CARVAllo, E. \& Del SOL, M. Int. J. Morphol., 35(1):368-370, 2017.

SUMMARY: The digastric muscle appears mentioned in Terminologia Anatomica with the code A04.2.03.006, it belongs to the group of the suprahyoid muscles and is formed by two bellys (latin: gaster) joined by an intermediate tendon. The anterior belly originates in the digastric fossa of the mandible and posterior belly in the mastoid notch of the tempral bone, both are inserted through a fibrous band, derived from the pretracheal layer of the deep cervical fascia on the body and greater horn of hyoid bone. Anterior and posterior bellys originates from the first and second arc gill respectively, the anterior belly being innervated by the mylohyoid nerve and the posterior belly by the facial nerve. In its name, the digastric muscle refers to the origin of the anterior belly and to the muscular classification according to form, not mentioning the origin of its posterior belly. The aim of this study was to review this term and recommend including in the name the "mastoid" origin of the digastric muscle, denominating it digastricomastoid muscle. This change is based on the different embryological origin and innervation of both bellys and is related to the objectives proposed by Terminologia Anatomica, which recommends descriptive, informative and harmonic names with related bone structures, favoring scientific communication and teaching-learning morphology

KEY WORDS : Digastric Muscle; Digastricomastoid Muscle; Terminologia Anatomica.

\section{REFERENCIAS BIBLIOGRÁFICAS}

Bergman, R. A.; Afifi, A. K. \& Miyauchi, R. Digastricus (Anterior belly). Digastricus (Posterior belly). En: Bergman, R. A.; Afifi, A. K. \& Miyauchi, R. Illustrated Encyclopedia of Human Anatomic Variation. Muscular System, Opus I. Anatomy Atlases, 2017. Disponible en: http:/ /www.anatomyatlases.org/Anatomic Variants/MuscularSystem/ Alphabetical/D.shtml 
Cabré Castellví, M. T. Theories of terminology. Their description, prescription and explanation. Terminology, 9(2):163-99, 2003.

Das, S.; Ghafar, N. A.; Othman, F.; Kamaruddin, A. \& Suhaimi, F. H. Anomalous belly of digastric muscle: Gross anatomy with clinical implications. Middle East J. Sci. Res., 21(5):733-5, 2014.

Edo Marza, N. Lexicografía especializada y lenguajes de especialidad: Fundamentos teóricos y metodológicos para la elaboración de diccionarios especializados. Lingüística., 27(1):98-114, 2012.

Jiménez Gutiérrez, I. La sinonimia y la polisemia en la terminología anatómica: Términos de ubicación y de relación de estructura anatómicas. Entreculturas, 1:579-97,2009.

Kalniev, M.; Krastev, D.; Krastev, N.; Vidinov; K.; Veltchev, L.; Apostolov, A. \& Mileva, M. A rare variation of the digastric muscle. Clujul Med., 86(4):327-9, 2013.

Liquidate, B. M.; Barros, D. M.; Alves, L. A. \& Barbosa, S. C. Anatomical study of the digastric muscle: Variations in the anterior belly. Int. J. Morphol., 25(4):797-800, 2007.

Mascaro, M. B.; Picoli, L. C.; Santos, F. M.; Bonsi, A. B.; Souza, M. R. \& Prosdócimi, F. C. Anatomical variation of the anterior belly of the digastric muscle: case report and clinical implications. J. Morphol. Sci., 28(1):72-5, 2011

Moore, K. L. \& Dalley, A. Anatomía con Orientación Clínica. $4^{\mathrm{a}}$ ed. Madrid, Editorial Médica Panamericana, 2007.

Platzer, W. Atlas de Anatomía. Con correlación clínica. Vol. I. Aparato Locomotor. $9^{\mathrm{a}}$ ed. Madrid, Editorial Médica Panamericana, 2008.

Rouvière, H. \& Delmas, A. Anatomía Humana: Descriptiva, Topográfica y Funcional. Vol. II. 10 a ed. Barcelona, Masson, 2002.

Testut, L. \& Latarjet, A. Tratado de Anatomía Topográfica. Tomo I. $8^{\mathrm{a}}$ ed. Barcelona, Salvat, 1979.

Whitmore, I. Terminologia Anatomica: new terminology for the new anatomist. Anat. Rec., 257(2):50-3, 1999.

\author{
Dirección de correspondencia: \\ Pamela Carvallo Semler \\ Universidad Católica de Temuco \\ Manuel Montt 56 \\ Temuco \\ CHILE
}

Email:pcarvallosemler@yahoo.es

Recibido : 19-10-2016

Aceptado :12-12-2016 